\title{
Large positive magnetoresistance in nonstoichiometric NiMnSb thin films on silicon
}

\author{
W. R. Branford, S. K. Clowes, M. H. Syed, and Y. V. Bugoslavsky \\ Blackett Laboratory, Imperial College, Prince Consort Road, London, SW7 2BZ, United Kingdom \\ S. Gardelis, J. Androulakis, and J. Giapintzakis \\ Foundation for Research and Technology-Hellas, Institute of Electronic Structure and Laser, \\ P.O. Box 1527, Vasilika Vouton, 71110 Heraklion, Crete, Greece \\ C. E. A. Grigorescu \\ National Institute for Research and Development for Optoelectronics, Bucharest, Romania
}

\author{
A. V. Berenov \\ Department of Materials Science, University of Cambridge, Pembroke Street, Cambridge CB2 3QZ, \\ United Kingdom \\ S. B. Roy \\ Low Temperature Physics Laboratory, Centre for Advanced Technology, Indore 452013, India \\ L. F. Cohen ${ }^{\text {a) }}$ \\ Blackett Laboratory, Imperial College, Prince Consort Road, London, SW7 2BZ, United Kingdom
}

(Received 3 November 2003; accepted 4 February 2004)

\begin{abstract}
We report a systematic study of the transport properties of pulsed-laser-deposited NiMnSb films on silicon as a function of film thickness. A low-temperature upturn is observed in the resistivity for film thicknesses of $130 \mathrm{~nm}$ and below. The resistivity minimum corresponds to the maximum in the positive magnetoresistance for all samples. As the film thickness decreases, the magnitude of both the resistivity upturn and the magnetoresistance increase. There is no feature associated with the upturn in the low-field Hall resistivity, which becomes systematically more electron dominated as the film thickness decreases and the temperature increases. This has implications for the use of $\mathrm{NiMnSb}$ as a spin injector for spintronic applications. The positive magnetoresistance of the $5 \mathrm{~nm}$ sample is greater than $100 \%$ at $200 \mathrm{~K}$ in $8 \mathrm{~T}$. Further enhancement of the magnetoresistance occurs for field parallel, rather than perpendicular, to the film surface. The magnetoresistance behavior is compared to various model systems, including the band-gap tuning found in the silver chalcogenides, disorder-induced weak localization, and the emerging class of "bad metal" ferromagnets. () 2004 American Institute of Physics. [DOI: 10.1063/1.1691172]
\end{abstract}

The Heusler ${ }^{1}$ alloy NiMnSb has attracted a great deal of attention as a potential material for spintronic devices, ${ }^{2,3}$ because in theory it is a half-metallic ferromagnet ${ }^{4}$ (HMF) with a Curie temperature $\left(T_{C}\right)$ of $728 \mathrm{~K},{ }^{5}$ and a saturation magnetization $\left(M_{S}\right)$ of $4 \mu_{B} /$ f.u. Hybrid spintronic devices require the growth of ferromagnetic films onto semiconductors for spin injection. The choice of semiconductor is governed by whether mobility or spin lifetime is maximized; narrowgap III-V's (Ref. 6) seem promising in the former case, and Si (Ref. 7) in the latter. However, the NiMnSb:semiconductor system is challenging to work with because (i) transport spin polarization ${ }^{8}\left(P_{t}\right)$ drops dramatically with a few percent atomic disorder, ${ }^{9}$ (ii) $P_{t}$ drops at the free surface due to surface reconstruction, ${ }^{10,11}$ and (iii) it has been reported ${ }^{12}$ that the true half-metallic state in NiMnSb only persists up to 80 K. Optimizing properties, particularly $M_{S}$ and $P_{t}$, is critical to the realization of hybrid spintronic devices.

The drive to continually increase data storage densities has sustained interest in magnetoresistance (MR) materials, particularly giant MR multilayers ${ }^{13}$ and colossal MR manganites. ${ }^{14}$ In these materials the magnetic field $(H)$ aligns

${ }^{a)}$ Electronic mail: 1.cohen@imperial.ac.uk the magnetic moments, which reduces the resistance. This is termed "negative magnetoresistance." Recently, magnetoresistances of comparable, ${ }^{15}$ or greater, ${ }^{16}$ magnitude to the CMR materials but opposite (positive) sign have been reported. In composite heterostructures ${ }^{16}$ the MR is a geometric effect, caused by magnetic deflection of the current path. In the nonmagnetic silver chalcogenides, ${ }^{15,17} \operatorname{Ag}_{2 \pm \delta} \mathrm{X}(\mathrm{X}$ $=\mathrm{Se}, \mathrm{Te})$, the magnetic field increases the intrinsic resistivity of the material through band-gap tuning, ${ }^{18,19}$ with the maximum in the magnetoresistance, and a linear field dependence, found at the crossover from hole-dominated to electron-dominated transport. In doped FeSi ferromagnets, ${ }^{20}$ the positive MR is associated with weak localization ${ }^{21,22}$ in disordered metals. In Ti:Si films, ${ }^{23}$ the positive MR vanishes below the sharp resistivity minimum.

Recently, it has been shown ${ }^{24}$ that a number of metallic Heusler alloys show a low temperature upturn in the resistivity, and are best described as strongly disordered electron systems, rather than conventional metals. Here we report the observation of a positive giant magnetoresistance in thin films of nonstoichiometric NiMnSb that is coupled to a lowtemperature upturn in the resistivity. The effect has a similar field dependence to the silver chalcogenides ${ }^{18,19}$ and the 


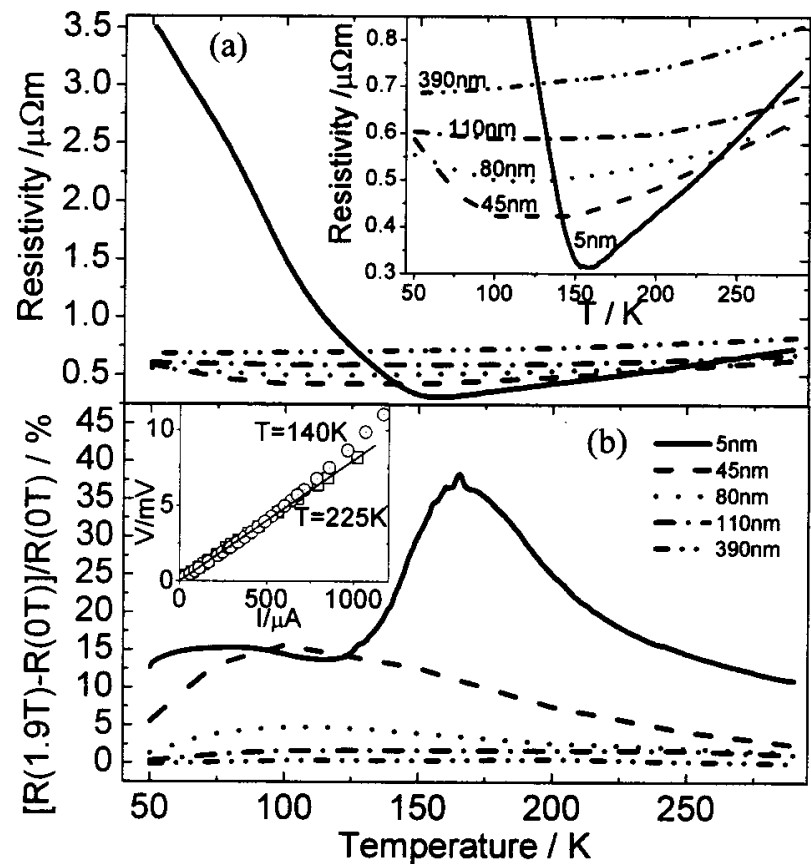

FIG. 1. (a) Zero-field resistivity vs temperature for NiMnSb films of different thicknesses. Inset: same data, enlarged in low resistivity region. (b) Magnetoresistance in $1.9 \mathrm{~T}$ as a function of temperature for series of films. Inset: $I-V$ curve of $5 \mathrm{~nm}$ film at 140 and $225 \mathrm{~K}$.

disorder-induced weak localization model. ${ }^{21,22}$

Thin films of NiMnSb, with thicknesses of 5, 45, 80, 110 , and $400 \mathrm{~nm}$, were grown on $\mathrm{Si}(100)$ by pulsed laser deposition ${ }^{6}$ at $200{ }^{\circ} \mathrm{C}$ from a slightly manganese-poor $\left(\mathrm{NiMn}_{0.95 \pm 0.01} \mathrm{Sb}\right)$ target. All films were shown by energy dispersive $\mathrm{x}$-ray analysis to be nonstoichiometric, formulated $\mathrm{Ni}_{1+x} \mathrm{Mn}_{1-y} \mathrm{Sb},(x=0.15 \pm 0.05, y=0.15 \pm 0.05)$. There was no systematic variation of stoichiometry between films of different thickness. The x-ray diffraction (XRD) pattern was consistent with a NiMnSb half-Heusler phase with the lattice parameter $5.99 \AA \pm 0.02 \AA$, compared to $5.9320 \AA$ $\pm 0.0028 \AA$ for the target, and no second phase was observed. The magnetic properties of the 45,110 , and $390 \mathrm{~nm}$ films were measured from 5 to $300 \mathrm{~K}$ in a Quantum Design SQUID magnetometer (a reliable signal could not be obtained for the $5 \mathrm{~nm}$ film). These three films had saturation magnetizations $\left(M_{S} \sim 2 \mu_{B} /\right.$ f.u. $)$ and the Curie temperatures were significantly reduced from the target $\left(M_{S}\right.$ $\sim 3.2 \mu_{B}$ /f.u.; $T_{C}=744 \mathrm{~K}$ ).

Magnetotransport data was collected in a van der Pauw geometry. The zero-field resistivity of the series of films is shown in Fig. 1(a), which is dominated by the sharp upturn in the resistivity of the $5 \mathrm{~nm}$ sample below $150 \mathrm{~K}$. The inset shows the temperature dependence of the other films. The resistivity upturn systematically decreases with increasing film thickness, and the $390 \mathrm{~nm}$ film does not show an upturn. In the thickest film the resistance ratio is $[\rho(290 \mathrm{~K}) / \rho(50 \mathrm{~K})=1.2]$. This shows that the transport is still inequivalent to bulk polycrystals, ${ }^{12}$ where $[\rho(290 \mathrm{~K}) / \rho(50 \mathrm{~K})=2.5]$ and the MR is negative. The temperature dependence of the MR, defined as $[R(H)$ $-R(0 \mathrm{~T})] / R(0 \mathrm{~T})$, in an applied field of $1.9 \mathrm{~T}$, perpendicular to the film surface, is plotted in Fig. 1(b). For all the films the MR is positive, with a maximum coincident in temperature Downloaded 03 Jun 2009 to 131.227.178.130. Redistribution subje

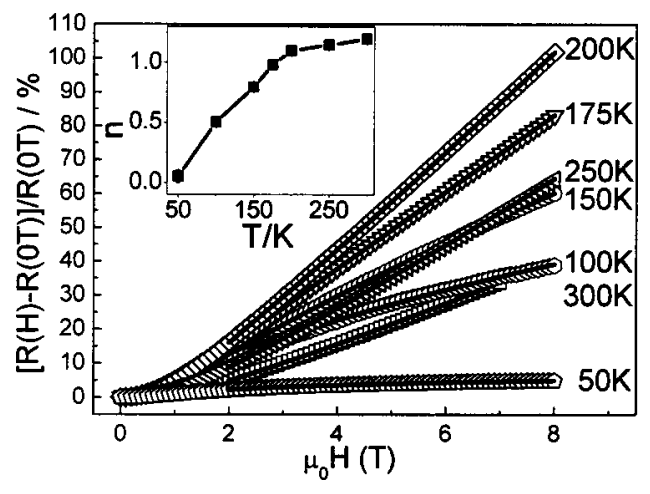

FIG. 2. Magnetoresistance of $5 \mathrm{~nm}$ film at selected temperatures (open symbols) and fit of high field $(B>2 \mathrm{~T})$ data to $\mathrm{MR}=\mathrm{MR}_{0}+a B^{n}$ (solid line). Inset: exponent $n$ obtained from this fitting procedure.

and minimum in the resistivity. The $390 \mathrm{~nm}$ film is an exception because it shows a broad maximum in the MR of $+0.5 \%$ around $150 \mathrm{~K}$, but no minimum is observed in the resistivity. The inset to Fig. 1(b) shows $I(V)$ curves of the 5 $\mathrm{nm}$ film, taken at temperatures with the same resistance on either side of the resistivity minimum, 140 and $225 \mathrm{~K}$. Below the resistivity upturn, the $I(V)$ curve deviates from Ohm's law for currents above $500 \mu \mathrm{A}$, indicating a profound change in the transport mechanism below the upturn.

To test whether anisotropic magnetoresistance (AMR) plays a significant role, the dependence of the large MR on field orientation was measured. When the field was applied parallel to the film surface, the MR was found to be slightly enhanced relative to the perpendicular MR, and was not affected by whether the current was perpendicular or parallel to the field direction. Hence, AMR does not significantly contribute.

The high-field and low-temperature behavior of the MR of the $5 \mathrm{~nm}$ sample is shown in Fig. 2. It should be noted that this data was collected with fresh contacts on a different instrument, and does not correspond exactly with those shown in Figs. 1 and 2. The field dependence of the MR has two regimes. In the low-field regime it varies as $H^{2}$ at all temperatures, whereas in the high-field regime the field dependence is clearly changing with temperature. The solid lines in Fig. 2 show a fit of the high field $(H>2 \mathrm{~T})$ data to $\mathrm{MR}=\mathrm{MR}_{0}+a H^{n}$ and the inset shows the fitted value of exponent $n$ as a function of temperature. The exponent is less than unity at temperatures below the resistivity upturn, and greater than unity above the upturn. The maximum in the MR occurs at the upturn temperature, where $n=1$.

Between 50 and $150 \mathrm{~K}$, the field dependence of the MR is superficially similar to the disorder-induced weak localization model. ${ }^{21,22,25}$ However, this model predicts an $H^{\frac{1}{2}}$ dependence in the high field limit, and $H^{2}$ at low field, with the theoretical crossover point around $H=\mathrm{kT} / g \mu_{B}$ which corresponds to $H=75 \mathrm{~T}$ at $100 \mathrm{~K}$ (assuming $g=2$ ). The fit of the $100 \mathrm{~K}$ MR with $n=1 / 2$ for $H \geqslant 2 \mathrm{~T}$ (Fig. 2) shows that the crossover occurs at around $2 \mathrm{~T}$. Furthermore, the plot of the exponent with temperature in the inset to Fig. 2 shows that the exponent of $H$ is slowly increasing with temperature, and is coincidentally close to $H^{1 / 2}$ around $100 \mathrm{~K}$. The model cannot predict either the crossover field or the temperature dependence of the exponent, and seems inappropriate for this to AIP license or copyright; see http://apl.aip.org/apl/copyright.jsp 


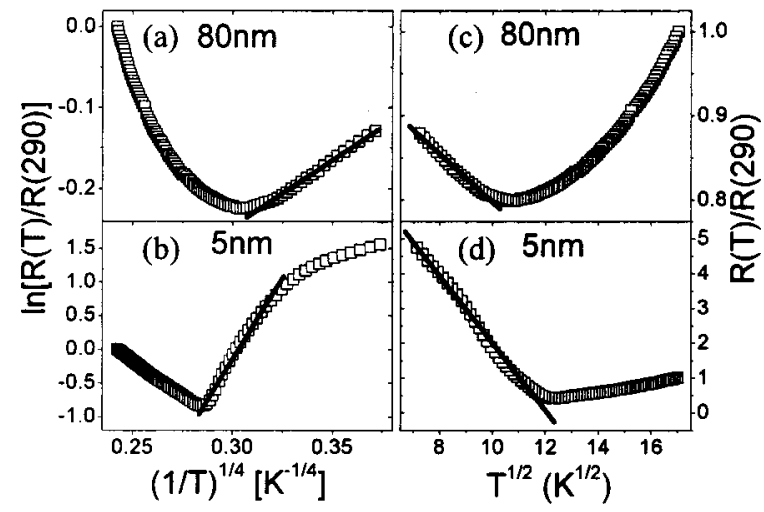

FIG. 3. Fit of low $T$ resistivity (see Ref. 28) of (a) $80 \mathrm{~nm}$ film at $100 \mathrm{~K}$ to variable range-hopping model, (b) $5 \mathrm{~nm}$ film at $100 \mathrm{~K}$ to variable rangehopping model, (c) $80 \mathrm{~nm}$ film at $100 \mathrm{~K}$ to weak localization model, and (d) $5 \mathrm{~nm}$ film at $100 \mathrm{~K}$ to weak localization model.

system, even if internal field enhancement ${ }^{20}$ of the applied field is considered.

The transition from sublinear to superlinear behavior of the MR with increasing temperature is similar to that seen in the silver chalcogenides, as is the observed ${ }^{19}$ maximum in the MR when the MR is linear in $H$. This linear, maximum MR was found to occur when the number of electrons and holes was equal, ${ }^{26,19}$ as demonstrated by a sign reversal in the Hall coefficient. The similarities between the NiMnSb films and the chalcogenides merit a Hall investigation. However, in $\mathrm{NiMnSb}$, the Hall analysis will not be transparent because of the anomalous Hall term, and the Fermi surface contains both holelike sheets and an electronlike sphere. ${ }^{4}$ Two-carrier Hall analysis is not straightforward. ${ }^{27}$

The MR is clearly linked to the low temperature upturn in the resistivity. The upturns in the 5 and $80 \mathrm{~nm}$ samples were fitted to a number of potential models, as has been reported ${ }^{28}$ for disordered $\mathrm{Ni}_{0.5} \mathrm{Al}_{0.5}$ alloys. The variable range hopping, weak localization, and Kondo mechanisms result in upturns that follow $(1 / T)^{1 / 4}, T^{1 / 2}$, and $\ln T$, respectively. This fitting procedure was not conclusive for these films, although the Kondo model was clearly inappropriate. Figure 3 shows fits to the other two models. The fit to the variable rangehopping model is shown for the 80 and $5 \mathrm{~nm}$ films in Figs. 3(a) and 3(b), respectively, and fits to the weak localization model for the 80 and $5 \mathrm{~nm}$ films in Figs. 3(c) and 3(d), respectively. The two models give reasonable fits of similar quality for the $80 \mathrm{~nm}$ sample. The $5 \mathrm{~nm}$ sample can be fitted reasonably with the $\left(T^{1 / 2}\right)$ weak localization model, and not at all with the $(1 / T)^{1 / 4}$ variable range-hopping model. Only the weak localization model gives a reasonable fit for both films. However, this model gave a poor description of the field dependence of the MR of the $5 \mathrm{~nm}$ sample. The upturn in the $5 \mathrm{~nm}$ sample is so much more dramatic than that of the other samples that it is not clear whether the same mechanism dominates the resistivity of all the films, or whether another mechanism is operating in the thinnest sample. Indeed nonlinearity in the $I-V$ curve of the $5 \mathrm{~nm}$ film below the upturn [inset to Fig. 1(b)] is suggestive of percolative behavior in this sample.
In summary, we report a giant positive magnetoresistance that increases with decreasing thickness in nonstoichiometric NiMnSb:Si films, and is greater than $100 \%$ for the 5 $\mathrm{nm}$ film at $200 \mathrm{~K}$ in $8 \mathrm{~T}$. The temperature of the maximum in the magnetoresistance corresponds to the minimum in the resistivity. There is a further, small enhancement of the MR when the field is applied parallel to the film surface. The evolution of the field dependence of the magnetoresistance appears similar to the silver chalcogenides, and is indicative of a crossover from hole-dominated to electron-dominated transport as the temperature increases.

The authors thank Mark Ellerby (UCL) for access to SQUID magnetometry and acknowledge the E.U. program G5RD-CT-2001 for funding.

${ }^{1}$ F. Heusler, Verh. Dtsch. Phys. Ges. 5, 219 (1903).

${ }^{2}$ S. Datta and B. Das, Appl. Phys. Lett. 56, 665 (1990).

${ }^{3}$ S. A. Wolf, D. D. Awschalom, R. A. Buhrman, J. M. Daughton, S. von Molnar, M. L. Roukes, A. Y. Chtchelkanova, and D. M. Treger, Science 294, 1488 (2001).

${ }^{4}$ R. A. de Groot, F. M. Mueller, P. G. van Engen, and K. H. J. Buschow, Phys. Rev. Lett. 50, 2024 (1983).

${ }^{5}$ M. J. Otto, H. Feil, R. A. M. Vanwoerden, J. Wijngaard, P. J. Vandervalk, C. F. Vanbruggen, and C. Haas, J. Magn. Magn. Mater. 70, 33 (1987).

${ }^{6}$ J. Giapintzakis, C. Grigorescu, A. Klini, A. Manousaki, V. Zorba, J. Androulakis, Z. Viskadourakis, and C. Fotakis, Appl. Phys. Lett. 80, 2716 (2002).

${ }^{7}$ Z. Wilamowski and W. Jantsch, J. Supercond. 16, 249 (2003).

${ }^{8}$ B. Nadgorny, 11. Mazin, M. Osofsky, R. J. Soulen, P. Broussard, R. M. Stroud, D. J. Singh, V. G. Harris, A. Arsenov, and Y. Mukovskii, Phys. Rev. B 63, 184433 (2001).

${ }^{9}$ D. Orgassa, H. Fujiwara, T. C. Schulthess, and W. H. Butler, Phys. Rev. B 60, 13237 (1999).

${ }^{10}$ D. Ristoiu, J. P. Nozieres, C. N. Borca, B. Borca, and P. A. Dowben, Appl. Phys. Lett. 76, 2349 (2000).

${ }^{11}$ G. A. de Wijs and R. A. de Groot, Phys. Rev. B 64, 020402 (2001).

${ }^{12}$ C. Hordequin, D. Ristoiu, L. Ranno, and J. Pierre, Eur. Phys. J. B 16, 287 (2000).

${ }^{13}$ M. N. Baibich, J. M. Broto, A. Fert, F. Nguyen Van Dau, F. Petroff, P. Etienne, G. Creuzet, A. Friedrich, and J. Chazelas, Phys. Rev. B 61, 2472 (1988).

${ }^{14}$ S. Jin, T. H. Tiefel, M. McCormack, R. A. Fastnacht, R. Ramesh, and L. H. Chen, Science 264, 413 (1994).

${ }^{15}$ R. Xu, A. Husmann, T. F. Rosenbaum, M. L. Saboungi, J. E. Enderby, and P. B. Littlewood, Nature (London) 390, 57 (1997).

${ }^{16}$ S. A. Solin, T. Thio, D. R. Hines, and J. J. Heremans, Science 289, 1530 (2000).

${ }^{17}$ M. M. Parish and P. B. Littlewood, Nature (London) 426, 162 (2003).

${ }^{18}$ A. A. Abrikosov, Europhys. Lett. 49, 789 (2000).

${ }^{19}$ M. Lee, T. F. Rosenbaum, M. L. Saboungi, and H. S. Schnyders, Phys. Rev. Lett. 88, 066602 (2002).

${ }^{20}$ N. Manyala, Y. Sidis, J. F. DiTusa, G. Aeppli, D. P. Young, and Z. Fisk, Nature (London) 404, 581 (2000)

${ }^{21}$ T. F. Rosenbaum, R. F. Milligan, G. A. Thomas, P. A. Lee, T. V. Ramakrishnan, R. N. Bhatt, K. Deconde, H. Hess, and T. Perry, Phys. Rev. Lett. 47, 1758 (1981).

${ }^{22}$ P. A. Lee and T. V. Ramakrishnan, Rev. Mod. Phys. 57, 287 (1985).

${ }^{23}$ M. F. Li and K. H. Wong, J. Magn. Magn. Mater. 197, 31 (1999).

${ }^{24}$ M. S. Lund, J. W. Dong, J. Lu, X. Y. Dong, C. J. Palmstrom, and C. Leighton, Appl. Phys. Lett. 80, 4798 (2002).

${ }^{25}$ P. A. Lee and T. V. Ramakrishnan, Phys. Rev. B 26, 4009 (1982).

${ }^{26}$ H. S. Schnyders, M. L. Saboungi, and T. F. Rosenbaum, Appl. Phys. Lett. 76, 1710 (2000).

${ }^{27}$ S. M. Watts, S. Wirth, S. von Molnar, A. Barry, and J. M. D. Coey, Phys. Rev. B 61, 9621 (2000).

${ }^{28}$ Y. P. Lee, Y. V. Kudryavtsev, V. V. Nemoshkalenko, J. Y. Rhee, and K. W. Kim, J. Appl. Phys. 91, 4364 (2002). 